Pecvnia, 10 (2010), pp. 95-124

\title{
La inconsistencia de la moral tributaria. El caso de los moralistas evasores
}

Recibido: Diciembre 2009 Aceptado: Febrero 2010

\author{
Victoria Giarrizzo \\ vgiarrizzo@gmail.com \\ Juan Sebastián Sivori \\ juansivori22@yahoo.com.ar \\ Universidad de Buenos Aires \\ Fac. de Ciencias Económicas \\ Córdoba 2120
}

Ciudad de Buenos Aires, Argentina C1120AAQ
La evasión tributaria es un fenómeno complejo. No existen causas ni soluciones únicas. Sin embargo, un factor determinante es la moral del contribuyente. Si bien el cumplimiento fiscal es un deber ciudadano, una de las cuestiones que se plantean cuando se explora la evasión en los países con incumplimiento tributario, es cuál es el grado de moral tributaria en esa sociedad, surgiendo preguntas como: ¿la evasión fiscal ocurre por la ausencia de moral tributaria o a pesar de la existencia de moral tributaria? Este trabajo explora la moral tributaria en la sociedad argentina, presentando evidencia sobre el grado de moral de los contribuyentes y destacando cómo un porcentaje mayoritario de la población entiende el incumplimiento fiscal como una acción incorrecta
The tax evasion is a complex phenomenon. There are neither single causes nor single solutions. Nevertheless, a determinant factor is the taxpayer's morality. Although the tax compliance is a civic duty, one of the questions that appears when the evasion is explored in the countries with substantial levels of evasion, is the degree of tax morality in those countries. Some questions arise such as: Does tax evasion happen for the absence of tax morality or spite of it? This paper explores the tax morality in Argentina, presenting evidence of a level of tax morality and emphasizing how an important part of the population understands evasion as an incorrect action, but even thought they evade. In so 
pero a pesar de ello, evade. Así, mientras la evidencia muestra que en los países con alto cumplimiento tributario la moral tributaria es elevada, no siempre ocurre el caso inverso. Sociedades con elevada moral tributaria pueden convivir con elevados niveles de evasión, irrumpiendo en escena un grupo de 'Moralistas Evasores'.

Palabras clave: evasión fiscal, impuestos, moral tributaria, incumplimiento tributario, moralistas evasores, informalidad. doing, while the evidence shows that in countries with high tax payment the tax morality is high, but it does not happen the other way round. Countries with high tax morality can coexist with high levels of evasion, appearing a group of 'Moralist Evaders'.

Key words: Tax evasion, taxes, tax morality, moralist evaders, moral compliance, informality.

\section{INTRODUCCIÓN}

En todas las economías del mundo, los Estados tienen la obligación de proveer a la sociedad un conjunto determinado de bienes y servicios públicos vinculados a la promoción del bienestar económico, social, ambiental y la redistribución de la riqueza. Para cumplir con estas responsabilidades, es necesario obtener niveles adecuados de ingresos fiscales. Por eso resulta crucial comprender mejor los múltiples inconvenientes que enfrentan los gobiernos al momento de recaudar impuestos de la población, un proceso arduo que en algunas economías suele derivar en tasas elevadas de incumplimiento e informalidad.

La evasión tributaria es un fenómeno complejo. No existen causas ni soluciones únicas, y si bien diversas teorías generales intentan abordarla, la forma en que se presenta en cada sociedad tiene características completamente idiosincrásicas. En países como Argentina, el incumplimiento fiscal se ha convertido a través de los años en un problema estructural, con consecuencias económicas y sociales muy graves, persistiendo un núcleo duro de evasión amplio, que no parece reaccionar ante los numerosos intentos realizados por el Estado: mayor fiscalización, castigos más severos, campañas de concienciación fiscal o premios para incentivar el pago. Si bien no hay cifras precisas, se estima que más de $35 \%$ de la economía argentina se encuentra en la informalidad, reflejándose esto en niveles elevados de precariedad en el mercado, que persisten incluso durante los ciclos económicos expansivos.

Pero aunque la evasión fiscal responde a causas múltiples, un factor determinante y al mismo tiempo escasamente explorado, es la 
moral del contribuyente. Es decir, cómo inciden (cuando inciden) los valores sociales, normativos o éticos de las personas en la decisión de pagar o evadir los tributos que le corresponden. Ya no se trata solo de la importancia que la sociedad le asigna al cumplimiento tributario, sino del nivel de gravedad que le asigna al no pago de sus impuestos, independientemente de la obligación normativa establecida en el marco legal.

Si bien el cumplimiento fiscal es un deber ciudadano y así suele ser entendido en las economías modernas por la mayoría de los individuos, el comportamiento frente al sistema tributario no siempre responde a lo que impone la norma, derivando en lo que habitualmente se denomina 'evasión'. Una de las cuestiones que se plantean cuando se explora la evasión en los países con niveles sustanciales de incumplimiento tributario, es cuál es el grado de moral tributaria en esa sociedad, surgiendo preguntas como: ¿la evasión fiscal ocurre por la ausencia de moral tributaria, es decir, por la carencia de normas o valores aceptados socialmente que establezcan que el no pago es algo incorrecto? ¿O el incumplimiento fiscal ocurre a pesar de la existencia de una moral tributaria?

La interacción entre moral y evasión tributaria presenta características singulares y particulares en cada país. Su estudio permite no solo entender con mayor profundidad las causas de la evasión, sino replantear el conjunto de mecanismos posibles para superarla. En economías como la Argentina, donde los niveles de evasión son relativamente elevados, la moral tributaria resulta en principio elevada pero presenta al mismo tiempo ciertas inconsistencias para explorar. La evidencia empírica recolectada, muestra que en términos de moral tributaria una población como la que se presenta en la sociedad argentina puede dividirse en tres segmentos bien diferenciados: un segmento conformado por individuos sin moral tributaria, otro conformado por individuos con moral tributaria débil, y un tercer segmento poblacional integrado por personas con moral tributaria fuerte. Si bien el comportamiento fiscal de cada uno de esos grupos se define por motivos completamente diferentes, surge un denominador común en ellos: la evasión es una característica casi permanente en los segmentos poblacionales que no manifiestan un vínculo entre moral y evasión, pero también se presenta con elevada frecuencia en los segmentos poblacionales donde sí existe una moral tributaria que permite que los hombres valoricen como algo 'incorrecto' el 'no pago de impuestos'. 
Uno de los principales hallazgos de esta investigación es destacar que un porcentaje mayoritario de la población en la Argentina suele entender al incumplimiento fiscal como una acción incorrecta y una falta a las responsabilidades civiles. Pero a pesar de ello, evade. Así, mientras la evidencia empírica muestra que en los países con alto cumplimiento tributario la moral tributaria es elevada, mostraremos en este trabajo que no siempre ocurre el caso inverso. Es decir, sociedades con elevada moral tributaria pueden convivir con elevados niveles de evasión, irrumpiendo en escena un grupo de 'Moralistas Evasores' en cuya moral conviven dos esquemas superpuestos e inconsistentes entre sí: la moral manifiesta y la moral efectiva. Es decir, dos esquemas donde las mismas personas que consideran incorrecto el no pago, si tienen la posibilidad de obtener algún beneficio económico por no pagar, eligen ese camino. Pero al mismo tiempo, y quizás lo más paradójico, es que 'no siempre sienten culpa de hacerlo', lo cual pone dudas sobre la interpretación más clásica de moral tributaria que establece que las normas sociales que guían la moral existen si el contribuyente siente culpa de evadir.

En términos de política económica, la identificación de grupos con diferentes grados de moral tributaria permite diseñar y definir políticas focalizadas para cada segmento. Pero tan relevante como conocer el nivel de moral tributaria de una sociedad, es analizar la consistencia de esa moral, esto es, hasta dónde esa moral manifiesta coincide con la moral efectiva de los individuos al momento de tomar decisiones relacionadas al cumplimiento fiscal. Así, las políticas para combatir la evasión posiblemente deban prestar mayor a atención a estos dos aspectos determinantes del grado de formalidad e informalidad de una economía para elaborar mecanismos que alienten el pago y desalienten la evasión.

El objetivo de este trabajo será explorar la moral tributaria, particularmente en la Argentina pero buscando obtener resultados generalizables. Para ello se identificarán los segmentos poblacionales según su nivel de moral tributaria, se analizarán las inconsistencias que se presentan en la dinámica evasión-moral, se evaluará por qué ocurren y finalmente se dejarán planteadas algunas rutas de abordaje posibles para enfrentar esta problemática, que trasciende las fronteras de lo meramente fiscal. 


\section{MORAL Y EVASIÓN EN LA LITERATURA ECONÓMICA}

Los estudios tradicionales sobre evasión tributaria estuvieron durante años centralizados en los aspectos coercitivos de la conducta fiscal. En esos modelos, el individuo decide evadir o pagar sus impuestos en función de la utilidad esperada de esa acción. La característica particular es que esa utilidad depende básicamente del ingreso esperado, la probabilidad de ser descubierto, el monto de la sanción, la tasa impositiva y la aversión al riesgo. Básicamente, estos estudios descansan en los modelos de toma de decisiones bajo incertidumbre, donde el individuo maximiza una función de utilidad sin que intervengan aspectos relacionados a la moral, la ética, u otras cuestiones vinculadas a las cargas valorativas de los contribuyentes. La decisión de evasión quedaría así representada por:

Utilidad Esperada de Evadir $($ UEV $)=$ Beneficio de evadir - costo de evadir

Utilidad Esperada de Pagar (UEP) = Beneficio de pagar - costo de pagar

El individuo decide evadir si: UEV $>$ UEP

Donde:

Costo de pagar $=$ valor del impuesto

Costo de evadir $=($ valor de la multa $) \times$ probabilidad de monitoreo

Si bien estos modelos concentraron durante décadas los análisis y estudios relacionados con la evasión fiscal, el enfoque tradicional comenzó a mostrar limitaciones cada vez más evidentes para explicar algunas cuestiones que parecían incidir significativamente en el comportamiento fiscal. Una pregunta que surgía con frecuencia era ipor qué aún en situaciones donde las penalidades por no pagar impuestos son bajas y las inspecciones poco frecuentes, algunas personas deciden igualmente pagarlos? ¿Por qué hay individuos que sienten culpa cuando no pagan y otros que no? La evidencia de que una proporción de los individuos simplemente no estaban predispuestos a evadir y el análisis de la influencia de otras variables como la moral o la percepción de justicia y equidad del sistema en la decisión de pagar o no los impuestos, fueron orientando las investigaciones hacia frentes alternativos y han permitido en las últimas décadas mejorar las teorías y modelos relacionados con la evasión tributaria. El cumplimiento o no cumplimiento fiscal dejó de ser visto solo como una función dependiente de variables objetivas como la probabilidad de ser detectado, la tasa tributaria o la penalidad impuesta, 
sino también ligado a la predisposición o voluntad íntima del individuo a pagar o no, que está determinada por un conjunto de factores subjetivos, como sus creencias, valores, o las normas sociales que le impone su entorno.

A partir de los años 90 la variable 'moral' comenzó a ser un factor comúnmente mencionado en la literatura económica cuando se analizan las causas que llevan a determinados contribuyentes a pagar sus tributos y a otros a evadirlos. Algunas aproximaciones teóricas y empíricas han intentando abordar la cuestión moral en la evasión tributaria, analizando aspectos como el sentido de culpa que puede percibir un individuo al evadir, el impacto de esa conducta en su reputación social, la influencia de la conducta del resto de los individuos, hasta dónde la evasión es considerada una conducta no ética o delictiva, o el grado de gravedad que el contribuyente le asigna a la evasión. Quizás una de las evidencias más fuertes que surgieron de estos estudios, fue comprobar que cuando en una sociedad el cumplimiento tributario es elevado, la moral tributaria también lo es, mientras que la ausencia de moral tributaria suele convivir con alto niveles de evasión.

de la moral

\section{II.1. Trabajos pioneros: primeras evidencias de la influencia}

Desde mediados del siglo pasado diversos autores han ido destacando que el cumplimiento fiscal y la evasión no pueden ser explicadas solo en función del nivel de penalidades, tasas y controles (Schmölders 1960, 1962, 1970; Kantona 1975; Graetz and Wilde 1985). Pero uno de los primeros trabajos donde se introduce la variable 'moral' en cuestiones vinculadas a la evasión y el cumplimiento tributario, es Allingham y Sandmo (1972), que curiosamente es considerado como el estudio seminal en temas de evasión fiscal. En "Income Tax Evasion: A Theoretical Analysis", los autores formalizan la actitud individual del contribuyente en su decisión de cumplir o no con sus obligaciones fiscales, introduciendo por primera vez un parámetro representativo del factor moral: la culpa por evadir y el impacto en su reputación social si es descubierto. Así, la maximización de la utilidad esperada de la evasión depende ya no solo del nivel de ingresos esperado en función de aquellas variables que determinaban la utilidad en el modelo tradicional que ellos mismos presentan, sino también de la 'culpa' por evadir que siente el individuo y del 'impacto en su reputación' que genera esa conducta. La conclusión del 
trabajo es que una mejor reputación, hace decrecer la utilidad marginal del ingreso por lo que reputación e ingreso serían sustitutos en sentido cardinal.

Una de las lecciones extraídas de este estudio, es que en sociedades donde la evasión afecta la reputación social del individuo, es de esperar que aumente el cumplimiento fiscal. Así, dentro de este modelo de utilidad esperada de la evasión, la moral tributaria sería un factor explicativo de por qué existen diferentes niveles de evasión en condiciones similares. Por ejemplo, dos personas con el mismo ingreso, necesidades económicas similares, la misma actividad, y la misma probabilidad de auditoría, pueden manifestar diferentes niveles de evasión si sus valores morales difieren. Si bien nada dicen esos modelos sobre cómo se instala una cultura tributaria social que relacione la evasión con la ética o la moral, sí aportan las primeras evidencias formales de que en la medida que la sociedad condena la evasión, o el individuo siente culpa de su conducta, el grado de incumplimiento tendería a reducirse.

En 1976, Spicer y Lundstedt avanzarán en la cuantificación de la incidencia de la reputación social en la actitud fiscal de los individuos. A partir de datos obtenidos en encuestas realizadas en Ohio, Estados Unidos, desarrollaron un modelo donde muestran que la decisión entre evadir o cumplir es un proceso de decisión que incluye otros conceptos, como la coercitividad, la equidad del sistema y la actitud de la sociedad hacia quienes no pagan. Para ellos, el problema de la evasión requiere entender los factores que motivan a los contribuyentes a incumplir. En la búsqueda de esas causas, en 1978, Song \& Yarbrought llevaron adelante la primera medición empírica en Carolina del Norte, EE.UU., para conocer la predisposición ética a las obligaciones fiscales de la sociedad, es decir, hasta dónde los individuos consideraban a la evasión como conducta delictiva o amoral. Sobre una muestra de 640 individuos, el $88 \%$ defendió el carácter delictivo de la evasión, pero sin embargo, no les resultaba mucho más serio que el robo de una bicicleta. A su vez, solo el $30 \%$ sentía la obligación de denunciar a los evasores frente a la autoridad. Por primera vez, aparece la asociación directa entre evasión y delito. Una característica del trabajo fue que los contribuyentes con mayores niveles de ingreso y mayor grado de educación fueron los que presentaron una mayor ética fiscal, en tanto quienes se sentían afectados por las políticas del Estado, mostraban una baja ética tributaria.

Desde entonces, múltiples trabajos abordaron la cuestión moral como un determinante esencial en el comportamiento fiscal. Weck 
(1983) a partir de un análisis econométrico, encuentra una correlación negativa entre la moral tributaria y la economía en negro (a mayor moral menor economía en negro y viceversa). Comparado con otras variables, la moral tributaria aparecía en esos estudios como la que mayor impacto tenía en la explicación de la economía informal. Otros trabajos anteriores Lewis $(1971,1982)$ indagan sobre la relación entre culpa, vergüenza y evasión, concluyendo que la culpa surge cuando los individuos comprenden que han actuado de manera irresponsable y violando una regla o norma social que ellos han internalizado como correcta. Aitken y Bonneville (1980) en una encuesta de opinión de contribuyentes encontraron que mas del $50 \%$ de los encuestados señalaron que sus conciencias se pueden ver afectadas después de hacer un conjunto de actividades, entre las cuales se encuentra el acto de evadir.

\section{II.2. Literatura reciente}

Desde aquellos trabajos pioneros, muchos autores han avanzado en el estudio de la moral tributaria, hasta que en 1994 finalmente Erard y Feinstein alientan a integrar los sentimientos morales en los modelos tradicionales para proporcionar una explicación razonable de comportamiento fiscal de los contribuyentes. Pero un abordaje especial surge por ese tiempo con el estudio de la interrelación entre la moral individual y la moral social. Reaparecen en esa línea de trabajo dos viejas teorías. Por un lado, la aproximación altruista (ya planteada por Chung en 1976) donde el individuo decide su comportamiento fiscal no solo en función de su propio bienestar sino en del bienestar social. Bajo este enfoque, la decisión de evadir o pagar está explicada por el conocimiento que tiene el individuo de que al no pagar se reduce la provisión de bienes y eso impacta sobre el bienestar social. Un segundo enfoque, es la aproximación kantiana de moral (ya planteada por Sugden en 1984) que está relacionada con la definición de Kant de moral, donde los contribuyentes consideran que un impuesto justo es aquel que es justo para todos los demás contribuyentes, y la evasión puede afectar su imagen frente a los demás.

En 1997, Bosco y Mittone realizan una diferenciación simple entre la moral en el sentido Kantiano, que se refiere a la reputación social de su conducta evasora, es decir, a cómo queda la imagen del evasor dentro de la sociedad, y la aproximación más altruista de la moral, que está vinculada al sentido de culpa o no culpa, que percibe el evasor 
por dejar de contribuir a la masa tributaria ya sea porque está infringiendo una norma o porque su conducta representa un perjuicio a toda la sociedad. La conclusión del trabajo es que para que un contribuyente decida evadir, los beneficios de hacerlo deben ser superiores a los costos morales que enfrenta, reafirmando con esto la idea original de Allingham y Sandmo. Sin embargo, un aspecto en el que reparan los autores, es que el costo moral solo aparecerá cuando los contribuyentes consideren que la tasa que se cobra es "justa", surgiendo acercamientos sobre algunos de los factores que inciden ya no necesariamente en la moral tributaria en sí, sino en el comportamiento del individuo a pesar de su moral tributaria.

Estudios recientes dan un paso más en ese análisis y dividen la moral en dos tipos, según de quien provenga. Por un lado, estaría la moral tributaria de los ciudadanos y por otro, la moral tributaria de quien formula el tributo, en general el Estado. Ambas "morales" interactúan y se transforman en una sola, a través de un "trade off" entre la imposición (por parte de quien la formula) y la aceptación (por parte de quien le recae). Estas dos morales correrían una carrera donde pueden surgir reformulaciones y reaceptaciones. Hasta que finalmente se llega a un acuerdo o consenso social sobre el tributo, donde el ciudadano acepta y legitima con su pago y rechaza con su no-pago o reclamo. Es en ese proceso donde se constituye una moral tributaria hegemónica, que adolecerá de consistencia cuando el nivel de rechazo sea constitutivamente alto.

En 2005, Torger and Martinez estudiaron la evolución de la moral tributaria en España como un factor determinante de la mejora en el cumplimiento tributario de este país. España constituye un caso de estudio interesante, porque logró que de un nivel de evasión del $40 \%$ registrado hacia fines de la década de los setenta, se llegara a que un $90 \%$ de los ciudadanos paguen sus impuestos. La pregunta que guió esta investigación fue ¿cómo hizo España para mejorar su moral tributaria en los últimos 30 años? Para ello se analizó la evolución ínter-temporal en la moral tributaria y la responsabilidad en las diferentes reformas institucionales que se fueron dando en ese país. Algunas de las conclusiones obtenidas, fueron que existieron al menos cuatro elementos centrales que cooperaron en una mejora de la moral tributaria. Por un lado, antes de la dictadura de Franco, la evasión recibía pequeñas sanciones. El primer cambio sustantivo en la moral tributaria ocurrió previo al Pacto de la Mondoa, donde la sociedad por primera vez en muchos 
años, percibió tener participación en la reforma tributaria. Esta mayor participación de la sociedad en la ley, que como eje principal tenía atacar a los grandes evasores, tuvo muy buena recepción en la sociedad mejorando significativamente la moral tributaria. Otro elemento determinante habría sido la entrada de España a la Comunidad Europea, que permitió homogenizar el sistema tributario Español con el resto de los países de la Comunidad, otorgando una mayor confianza institucional que también incidió positivamente en la moral tributaria. Un tercer factor fue la ampliación de un esquema de impuestos progresivos, que tuvieron una recepción "favorable" en los ciudadanos y actuaron positivamente sobre su moral tributaria. Por último, se destaca la etapa 1981- 1995, donde se lograron las mejoras más importantes en la moral tributaria, impulsadas por el crecimiento de la confianza, la adhesión a las políticas públicas tomadas por el gobierno y una mayor entendimiento de la población sobre cómo la contribución tributaria contribuye a mejorar el nivel del vida de la sociedad.

Actualmente, los estudios de evasión fiscal suelen coincidir en que la moral fiscal actúa como una motivación intrínseca para pagar impuestos, y puede ayudar a entender los diferentes niveles de evasión entre países o en un mismo país entre diferentes segmentos poblacionales (Lewis 1982; Roth et al. 1989; Alm et al. 1999; Frey 2003; Frey and Torgler 2002). Sin embargo, los estudios de moral tributaria constituyen un área donde hay mucho por desarrollar e investigar. La existencia de moral tributaria es una condición necesaria para reducir la evasión e incrementar el cumplimiento fiscal. Sin embargo, no parece ser una condición suficiente. La evidencia empírica muestra que en los países con alto cumplimiento tributario la moral tributaria es elevada. Sin embargo, arrojaremos evidencia en este trabajo donde no siempre ocurre el caso inverso. Es decir, sociedades con elevada moral tributaria pueden convivir con elevados niveles de evasión, surgiendo grupos de Moralistas Evasores cuya moral presenta un conjunto de inconsistencias notorias y relevantes.

\section{MORAL MANIFIESTA versus MORAL EFECTIVA}

La evidencia de que la cuestión moral es un determinante importante en el comportamiento fiscal de los contribuyentes parece fuerte y sólida. Sin embargo, si bien la literatura económica muestra que las sociedades con bajos niveles de evasión presentan elevados niveles de 
moral tributaria, una de las conclusiones que surgen en este trabajo es que esa relación no siempre ocurre en el orden inverso: en determinados países con niveles elevados de evasión tributaria, esa informalidad se presenta a pesar de registrarse niveles altos de moral tributaria. El problema surge en la inconsistencia visible de la moral tributaria de algunos contribuyentes, que da lugar a la conformación de grupos de Moralistas Evasores, que por diferentes razones, están dispuestos a violar sus propias normas y convicciones.

\section{III.1. Determinando la moral}

La determinación del grado de moral tributaria se puede realizar al menos de dos formas. Por un lado, analizando cómo valora el contribuyente la evasión en sí misma, es decir, el no pagar un impuesto cuando tiene la responsabilidad tributaria de hacerlo. La segunda, es analizando cómo valora la evasión el demandante de un bien o servicio que si bien no es el responsable directo de pagar el impuesto, con su conducta alienta el incumplimiento fiscal. Un ejemplo de este segundo caso podría ser cómo valora el 'acto de comprar en negro' el consumidor que adquiere un producto en el mercado informal. En este trabajo, elegimos esta segunda opción, tanto porque su sencillez metodológica permite obtener datos más confiables (al usar encuestas, los datos revelados por consumidores suelen ser más confiables y sencillos de recolectar que los revelados por empresarios), como porque del comportamiento indirecto del comprador se puede inferir mejor su moral, ya que el contribuyente al no ser el que evade directamente, contesta con mayor aproximación lo que piensa, sin interponer ese pensamiento con lo que sería políticamente correcto.

Cuando se explora la moral tributaria de los contribuyentes desde este enfoque, se pueden identificar al menos tres segmentos poblacionales bien diferenciados, según el grado de moral tributaria de cada uno de ellos. Estos son:

Segmento 1. Sin Moral Tributaria (SMT): es el porcentaje de la población que 'no' valora como algo incorrecto comprar en negro, ya sea porque cree que no está mal o porque desconoce si es una acción incorrecta.

Segmento 2. Moral Tributaria Débil (MTD): es el porcentaje de personas que valora como algo 'más o menos' incorrecto comprar en negro. 
Segmento 3. Moral Tributaria Fuerte (MTF): es el porcentaje de personas que 'sí' valoran como algo incorrecto comprar en negro.

Sobre los tres segmentos poblacionales establecidos en función de su nivel de moral tributaria, se puede esperar a priori niveles de evasión elevados en los segmentos 1 y 2 , donde la moral ausente o débil determina ese comportamiento fiscal. Esto confirmaría los estudios recientes (algunos mencionados en la Sección II), donde se marca que los bajos niveles de moral determinan elevados niveles de evasión.

Sin embargo, esta relación se vuelve difusa en el segmento 3. Si bien es de esperar que en este segmento los niveles de evasión sean bajos o nulos, esa relación no siempre parecería cumplirse. Es decir, no necesariamente personas que pertenecen al segmento 3 (Moral tributaria Fuerte) se comportan según los valores que manifiestan, apareciendo contradicciones en el 'deber' y el 'hacer', o, dicho de otra forma, surgiendo dos tipos de morales, la Moral Manifiesta y la Moral Efectiva del individuo. Eso deriva en conclusiones completamente diferentes a las que surgen del estudio de los segmentos 1 y 2, tanto al intentar explicar las causas de la evasión como en la propuesta de soluciones.

Nuestra primera hipótesis de trabajo es que en sociedades con determinadas características, es posible encontrar que la evasión ocurra tanto bajo la ausencia de moral tributaria, como en presencia de la misma, lo que deriva posiblemente en un problema de evasión mucho más profundo y difícil de erradicar. En la sección siguiente se presentará evidencia que corroboraría esta hipótesis, a partir de una exploración profunda de la moral tributaria en la argentina.

\section{III.2. La moral tributaria en la Argentina: ¿regla o excepción?}

El estudio de la moral tributaria se abordará en esta sección evaluando el comportamiento de los consumidores frente al Impuesto al Valor Agregado (IVA) en la Argentina, de acuerdo al segundo enfoque explicado en la sección III.1. El IVA es un impuesto indirecto sobre el consumo donde la empresa se lo cobra al cliente, pero luego deberá abonarlo al Estado. La esencia de este tributo es que los consumidores finales tienen la obligación de pagarlo y ese pago es controlado por el organismo fiscal a través del comprobante o factura que la empresa le entrega al cliente. Lo interesante de este abordaje, es que al no ser 
responsabilidad del consumidor el pago del tributo, no recibe castigos por comprar en negro, y eso permite esterilizar la variable 'moral', limpiando del análisis temas relacionados con el riesgo de ser descubierto y las multas correspondientes.

Para analizar la moral tributaria en la Argentina, se llevó adelante una encuesta entre 1.500 personas en Capital Federal y Gran Buenos Aires ${ }^{1}$. El relevamiento, realizado entre septiembre y octubre de 2009, se desarrolló en dos tramos. En el primero se buscó captar aspectos generales relacionados con la evasión, la responsabilidad de los consumidores y vendedores, sus hábitos, creencias y auto fundamentos. En el segundo, se indagó sobre cuestiones más directas relacionadas estrictamente con su moral tributaria, buscando fundamentos más íntimos, como la evaluación que hace la población al acto de comprar en negro (tanto cuando compran otros como cuando compran ellos mismos), cómo se considera a la evasión tributaria en sí misma, por qué motivos compran en negro, cuál es su grado de conciencia de que se está comprando informalmente, por qué hay reticencias a solicitar el comprobante al realizar una compra, si cree que pagar impuestos es una obligación, cómo es su comportamiento ético en la vida, entre otros aspectos. El objetivo fue, mediante encuestas, determinar cuestiones como:

a) ¿Cuál es el grado de moral tributaria de la sociedad argentina?

b) ¿Cómo está incidiendo esa moral sobre el pago o no pago de impuestos? ¿Mayor moral se condice con mejor cumplimiento fiscal?

Para una vez repasado el estado de la moral tributaria comenzar a determinar cuestiones como:

a) ¿Cuáles son las causas que llevan a una sociedad a tener un grado mayor de moral tributaria que otra?

b) ¿Qué puede hacer el Estado para mejorar la moral tributaria?

Las principales conclusiones y evidencia que surgieron de la encuesta realizada fueron:

Esta encuesta se focalizó en Capital Federal y Gran Buenos Aires, donde se concentra el $31,6 \%$ de la población del país. Pero constituye una primera etapa. El objetivo es extender la encuesta hacia otras localidades del país. Fue una encuesta directa, mediante formulario estructurado realizado a mayores de 18 años mediante método aleatorio. El diseño muestral fue probabilístico bieapico por conglomerados, estratificado por nivel de ingresos. 
- Comprar en negro: malo para unos, indiferente para otros...

Lo primero que se buscó determinar en la encuesta fue el estado de la moral tributaria en la Argentina. Para eso se eligió una pregunta muy simple: ¿Cree que comprar en negro está mal? La pregunta tenía cuatro opciones de respuestas: Sí; Más o Menos; No; No lo sé.

A partir de las respuestas obtenidas, se especificaron los tres segmentos poblacionales señalados en la sección III.1. A quienes consideraron que comprar en negro Sí es un comportamiento incorrecto se los consideró dentro del grupo de 'Moral Tributaria Fuerte' (MTF); a quienes consideraron que comprar en negro es Más o Menos incorrecto, se los ubicó dentro del segmento de Moral Tributaria Débil (MTD); y a quienes consideraron que No es una acción incorrecta o contestaron No sé, se les incluyó en el segmento poblacional 'Sin Moral Tributaria' (SMT). Así, en el caso de la Argentina, estos tres segmentos quedaron constituidos de la siguiente forma:

Segmento 1. Sin Moral Tributaria: el 23,4\% de la población manifestó ausencia de moral tributaria, ya sea porque no considera que comprar en negro sea algo incorrecto o porque desconoce si es malo o bueno.

Segmento 2. Moral Tributaria Débil: el 17,1\% de la población argentina tiene una moral tributaria débil y solo valoró como algo 'más o menos' incorrecto comprar en negro.

Segmento 3. Moral Tributaria Fuerte: el 59,5\% de la población parecería contar con una moral tributaria fuerte, al considerar como algo incorrecto el acto de comprar en negro.

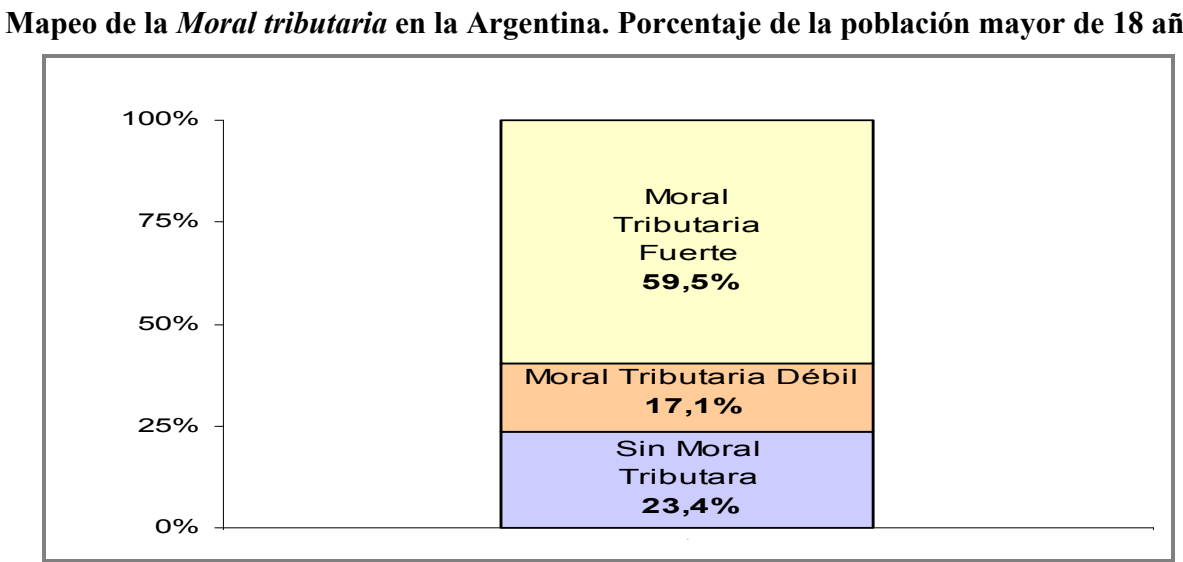


- Es importante pedir el comprobante, pero no lo pido...

El IVA es uno de los impuestos de mayor peso en la recaudación argentina: explica casi $30 \%$ de lo que recauda el Estado Nacional. Una característica de ese tributo, es que el consumidor puede ejercer presión sobre el contribuyente responsable de pagarlo (la empresa) solicitando el comprobante luego de realizar la compra. Sin embargo, eso no ocurre: a pesar de las campañas de concienciación y programas de aliento que frecuentemente relanza el ente controlador (la Administración Federal de Ingresos Públicos - AFIP) para incentivar a la población a pedir el comprobante de compra, la mayor parte de los consumidores en la Argentina no piden su factura. De acuerdo con la encuesta, solo $20,3 \%$ de los encuestados manifestó que pide siempre el ticket, $25,0 \%$ no lo pide nunca, y el resto, dependiendo el comercio o el tipo de producto o servicio que se adquiere.

La pregunta que surge inmediatamente es: ¿no se pide el ticket porque no se considera importante hacerlo? Los resultados sorprenden: para la mayoría de la gente $(71,8 \%)$ es importante pedir el ticket, para el resto $(28,2 \%)$ es solo medianamente importante o directamente no es importante ${ }^{2}$.

Si al realizar un consumo, el comercio no le entrega ticket ¿Lo pide?

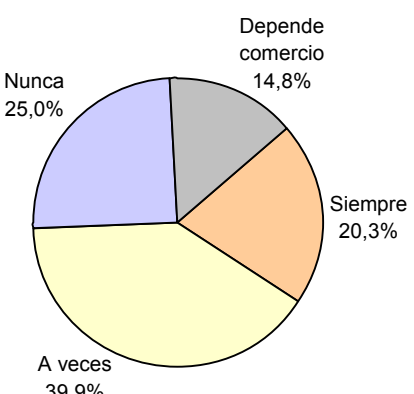

$39,9 \%$
¿Le parece importante pedir el ticket?

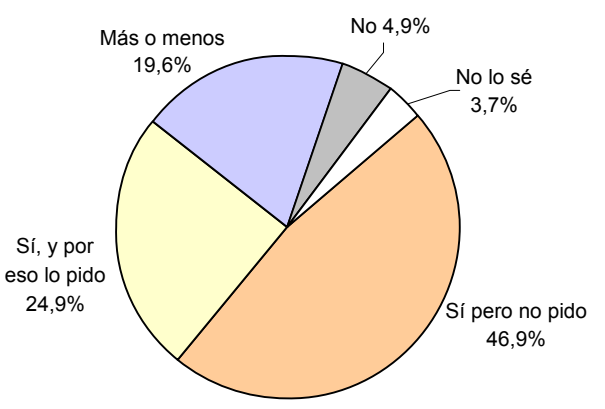

El porcentaje de la población que respondió 'no lo se' $(4,1 \%)$ se incluyó dentro de la población para la cual la solicitud del ticket no es importante. En este caso, la no importancia queda inducida por el desconocimiento que tiene el consumidor de lo que implica impositivamente obtener el comprobante. 
Es decir, el $71,8 \%$ de los encuestados declara que es importante pedir el ticket, sin embargo, a pesar de ello, el 79,7\% de la gente no lo pide.

En principio, se pueden argumentar dos puntos relevantes, uno positivo y uno negativo:

1) La población que reclama siempre su ticket $(20,3 \%)$ representa el control efectivo a la evasión fiscal que realizan los consumidores, que, como se puede observar, es muy bajo.

2) Hay un $28,2 \%$ de la población (de la población encuestada) que le asigna importancia baja o nula al acto de pedir el comprobante. Sobre este segmento poblacional las autoridades económicas deberían trabajar introduciendo educación tributaria e instalando mayor conocimiento y conciencia sobre la importancia e implicaciones que conlleva obtener el comprobante de compra una vez efectuada la transacción. Ya no pasa por el hábito de pedir o no pedir el comprobante de compra, sino porque directamente para ese segmento de la población el comprobante no es algo significativo.

Ese $28,2 \%$ que no le asigna importancia al ticket de compra podría ser interpretado como un piso de la evasión en la Argentina, la población perteneciente al segmento poblacional 1 (SMT) para la cual comprar en blanco o en negro es lo mismo. Sin embargo, esto no sería tan lineal, ya que la misma encuesta revela que un porcentaje no menor de la población no relaciona la no entrega del ticket de un comercio con 'comprar en negro' $(22,6 \%$ no considera que cuando no le dan ticket está comprando en negro y otro $29,2 \%$ sostiene que a veces sí y a veces no). A su vez, tampoco puede saberse mediante ese tipo de preguntas lo que sucede con la población de los segmentos 2 y 3 , ya que quienes sí creen que es importante pedir ticket no siempre lo relaciona con algún tema tributario: muchas personas consideran que es importante pedir el comprobante solo por si necesitan cambiar el producto o reclamar el servicio.

Indagando con mayor profundidad sobre los motivos por los cuales no se pide el comprobante, la principal razón argumentada es 'por no esperar' $(78,8 \%$ de las respuestas). 
¿Por qué no solicita el ticket?

(calculado sobre el \% de la población que no pide siempre el comprobante)

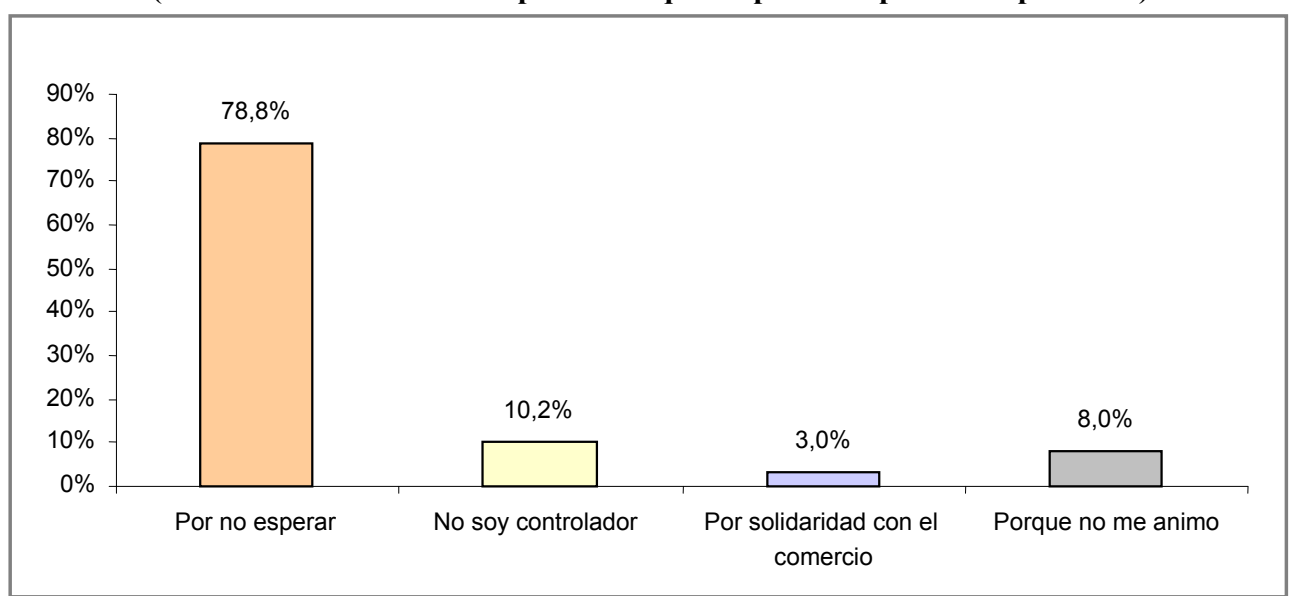

Una vez testeada la actitud del consumidor frente al control indirecto que puede ejercer en el IVA (pidiendo o no el comprobante), se buscó determinar en forma más directa cómo evalúa el individuo la actitud del vendedor cuando no le entrega el comprobante, cómo actúa frente a la posibilidad de obtener cierto beneficio económico haciéndose cómplice del vendedor (aceptando comprar en negro) y cuáles son sus valoraciones generales sobre sus obligaciones tributarias.

- Evadir es incorrecto, pero cuando se me presenta la oportunidad, ¿evado?...

Para analizar cuál es la evaluación que realiza el consumidor sobre la evasión se realizaron una serie de preguntas de orden diverso. Algunas de estas preguntas fueron:

a) ¿Cree que pagar impuestos es una obligación?

b) Cuando compra un producto y el comerciante no le entrega ticket, ¿cree que eso es incorrecto?

c) ¿Cree que comprar en negro está mal?

d) ¿Cree que la evasión tributaria es algo incorrecto?

e) Si al realizar una compra de $\$ 121$ el comerciante le da a elegir entre: 1. Pagar $\$ 121$ y llevarse el ticket; 2 . Pagar $\$ 110$ pero no llevar el ticket, ¿Qué elige? 
Las respuestas fueron las siguientes:

- El 86,0\% concuerda en que pagar impuestos es una obligación

- El 79,7\% señala que es incorrecto no recibir factura de compra. Este porcentaje coincide con bastante aproximación con la cantidad de personas que consideran importante pedir ticket $(72,3 \%)$

- El 59,5\% cree que comprar en negro está mal

- El 78,8\% sostiene que la evasión tributaria es algo incorrecto
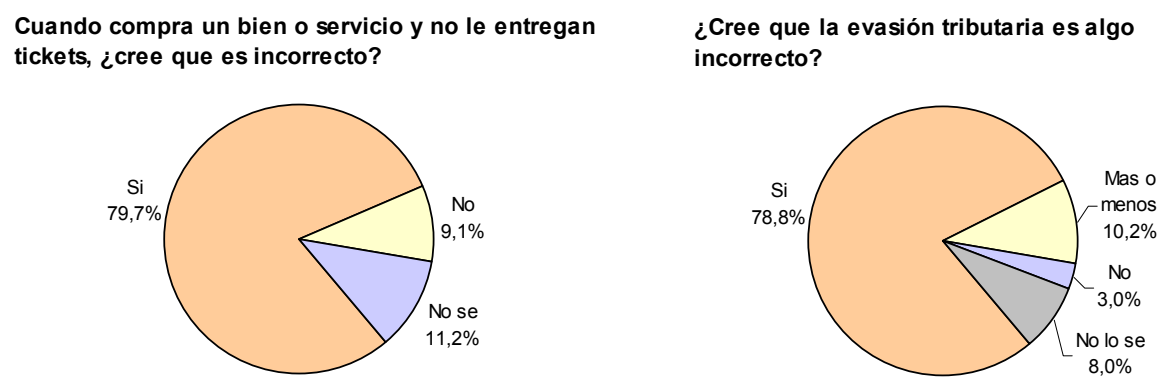

¿Considera que pagar los impuestos que corresponden Si al realizar una compra de $\$ 121$ el comercio le es una obligación?

$$
\text { da a elegir: 1. Pagar } \$ 121 \text { y llevar el tickets; } 2 \text {. }
$$

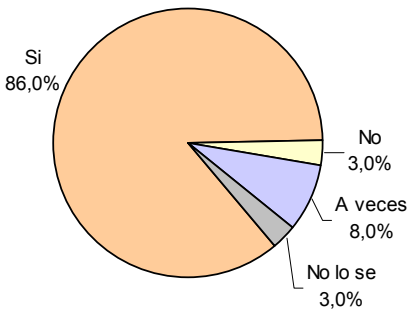
Pagar $\$ 110$ sin llevar tickets, ¿Qué elige?

Es decir, la mayor parte de los individuos parecerían comprender que pagar impuestos es una obligación. Esta evidencia refuerza el concepto que denominamos Moral Manifiesta. Sin embargo, cuando existe la posibilidad de obtener un beneficio económico violando las reglas tributarias, la Moral Efectiva dice otra cosa: si el vendedor le diera a elegir al comprador entre pagar $\$ 121$ "en blanco" (con factura) o $\$ 110$ "en negro" (sin factura), el 58,6\% elige comprar "en negro" para ahorrarse $\$ 11$. Aquí aparece la primera contradicción de la gente: el $71,8 \%$ de los individuos dicen que es importante pedir ticket, a su vez el $79,7 \%$ declara que es 
incorrecto que no le entreguen el comprobante de pago, el $86,0 \%$ comprende que pagar impuestos es una obligación, $78,8 \%$ sostiene que la evasión tributaria es algo incorrecto y si se quiere una respuesta más directa, hasta $59,5 \%$ señala que comprar en negro está mal. Pero a pesar de manifestar la importancia de cumplir con las obligaciones fiscales, si pueden ahorrase $\$ 11$, el $58,6 \%$ elige dejar de lado sus valores morales y 'evadir'.

Claramente, el público parece responder al incentivo económico. Por un lado, declara que es importante pagar, pero si dadas las circunstancias, pueden ahorrarse dinero, las cuestiones relacionadas con la ética, la moral y lo legal, pasan a un segundo plano.

\section{- El moralista evasor}

Quizás el dato más relevante que surge del muestreo, sea el siguiente: el $42,5 \%$ de las personas del segmento 3 (moral tributaria fuerte) eligen comprar en negro por ahorrarse $\$ 11$. Es decir, el $42,5 \%$ de la gente con moral tributaria fuerte está dispuesto a dejar de lado sus valores morales y evadir si se antepone en esa decisión la posibilidad de obtener cierta ventaja económica. Aparece acá la figura del Moralista Evasor. Se trata de individuos con moral tributaria fuerte, pero que violan sus propias convicciones morales.

Lo que manifiesta la gente cuando le asigna importancia al pago de impuestos es su deber moral; aquello que es políticamente o moralmente correcto. No obstante, cuando realiza sus transacciones económicas se deja llevar por sus motivos más íntimos, por sus intereses económicos $\mathrm{y}$, dado que estos últimos son mas fuertes que el "deber moral", el individuo puede terminar evadiendo. En este caso, la contraposición es moral manifiesta ("es importante pagar") y moral efectiva ("si tengo beneficios económicos, decido no pagar").

- Acomodando la moral a la acción...

Una vez captada la valoración de los diferentes aspectos vinculados a la evasión y la compra informal, el cuestionario indujo al individuo a evaluar su propia acción. Lo primero que se buscó explorar es si el individuo que compra sin ticket es consciente de que pudo haber existido en esa transacción un acto de evasión. A la pregunta: Cuando 
compra un producto y no le dan ticket, ¿cree que está comprando en negro? Entre quienes eligieron pagar más barato (\$110) y no llevar el ticket para ahorrarse $\$ 11$, solo $27,8 \%$ consideran que no están comprando en negro. El resto $(72,2 \%)$ señalan que sí están comprando en negro (35,5\%), que 'a veces' (29\%) ó 7,7\% señalan 'no lo se'. Así, el 72,2\% manifiesta a través de su respuesta que pudo haber existido un acto de evasión en su compra. Con lo cual, se descarta así la hipótesis de que eligió pagar $\$ 110$ porque no sabía que con eso compraba en negro. A su vez, se reafirma la pregunta con otra: ¿cree que comprar en negro está mal? El 59,5\% contestó que sí, el $17,1 \%$ más o menos, determinando con esta pregunta los segmentos poblacionales según su grado de moral tributaria.

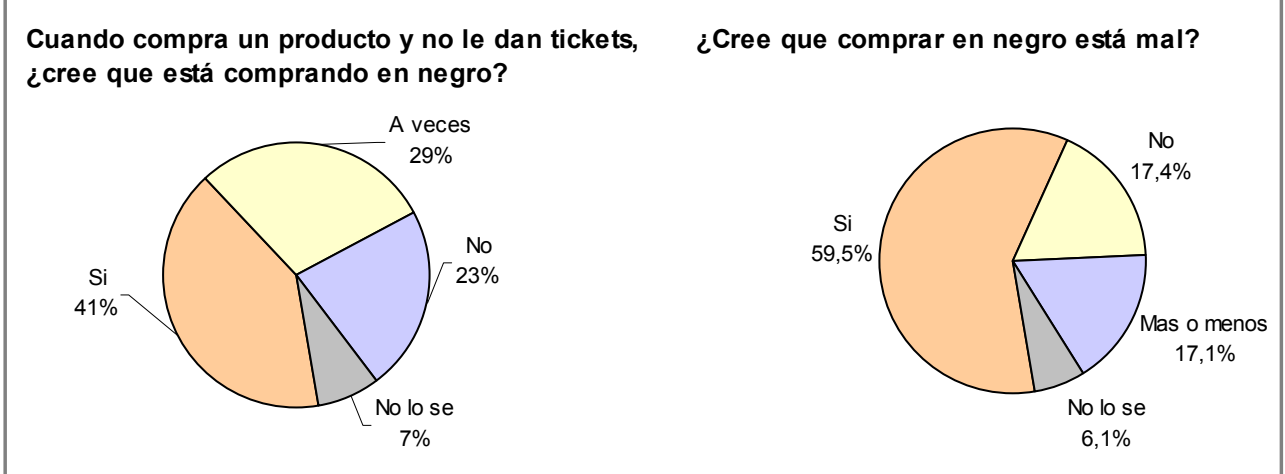

Sin embargo, esas respuestas encuentran sostén en la justificación de esa acción. Si bien la mayor parte de la gente cree que es incorrecto comprar en negro, cuando se refiere a sí mismo (comprar en negro para pagar menos), solo $27,3 \%$ considera que hizo algo incorrecto.

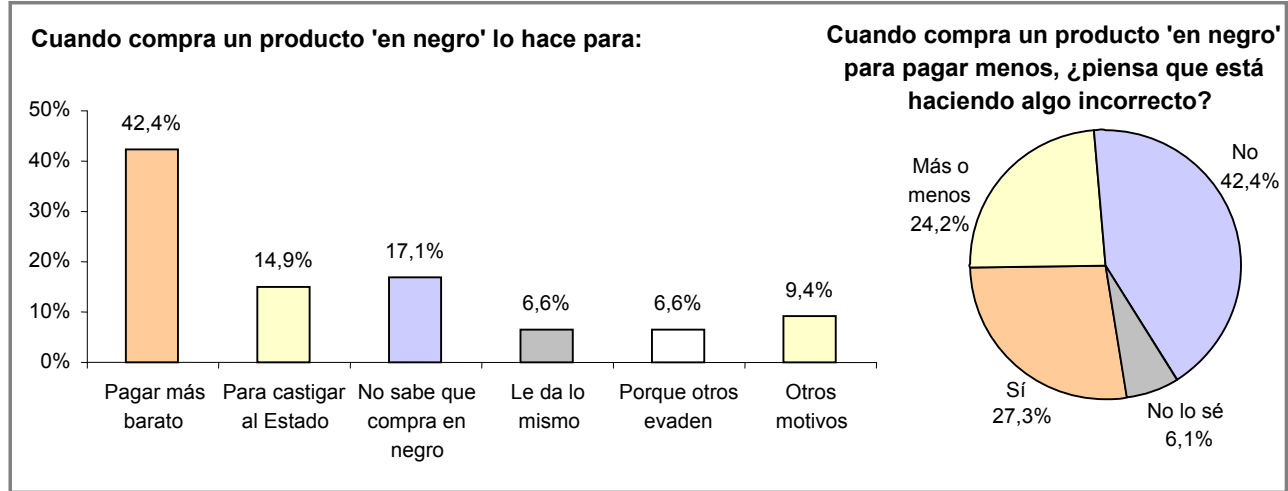


- Evado y sin culpa...

Un aspecto de la moral está relacionado con la culpa. Suele entenderse que las normas sociales que guían la moral en determinadas acciones existen siempre que el acto contrario a esas normas genere culpa en el individuo que lleva adelante la acción y vergüenza en el caso que fuera descubierta su conducta (Andreoni, Erard y Feinsten 1998). En el caso de la evasión, las normas sociales que guían la moral tributaria existirían si el individuo que evade siente luego culpa por su comportamiento. Sin embargo, ese no parece ser el caso en la sociedad argentina. Aunque la mayor parte de la población considera como algo 'incorrecto' la evasión, cuando evaden la mayoría de los individuos no parecen sentir 'culpa' o 'arrepentimiento' de sus actos.

Específicamente, a la pregunta: cuando compra un producto en negro para pagar menos, ¿siente culpa? El 67,3\% de los encuestados contesta la opción 'para nada'. Vuelve acá el tema de la 'Moral Manifiesta' versus la 'Moral Efectiva'. La gente sabe que está haciendo algo incorrecto pero no siente culpa de ello. Solo $9,2 \%$ dice sentir mucha culpa. La no culpa de la evasión se da con intensidad también en el segmento 3 , es decir, en el grupo poblacional con Moral tributaria fuerte. Y eso lleva a un tema central: ¿por qué a pesar de tener una moral tributaria fuerte, los individuos evaden sin culpa?

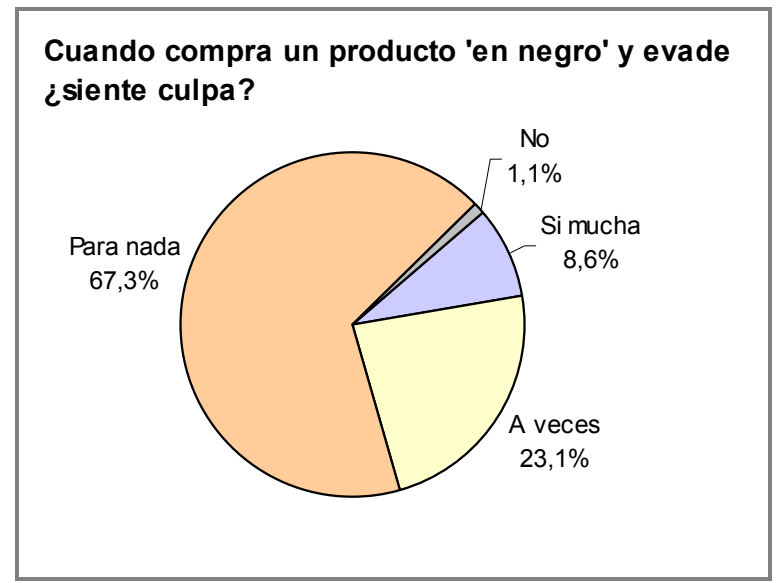

Posiblemente la 'no culpa' en la evasión tiene que ver con otras características de la economía. Por ejemplo, la percepción de niveles elevados de corrupción en el Estado, o de niveles de ingresos 
insuficientes que lo llevan a autojustificar su conducta fiscal. Tres preguntas que acompañaron a la encuesta fueron:

1. Si sus ingresos aumentaran, ¿pagaría más sus impuestos?: solo $31,3 \%$ de las respuestas fueron positivas, y $44,7 \%$ de respuestas negativas.

2. Si tuviera los ingresos suficientes para mantener el estándar de vida que quisiera, ¿pagaría todos sus impuestos?: el $72,2 \%$ de respuestas fueron positivas.

3. Si hubiera menos corrupción en el gobierno, ¿pagaría todos sus impuestos?: $61 \%$ de las respuestas positivas.

Los problemas de ingresos y la percepción sobre la corrupción del Estado parecen ser dos variables relacionadas no necesariamente con la moral tributaria manifiesta, pero sí con la moral tributaria efectiva. Los ingresos constituyen un impedimento al pago y en la evasión el público encuentra parte de su compensación por sus ingresos insuficientes. A su vez, la percepción de corrupción en el Estado autoriza a la población a no sentirse culpable por una acción a la cual el mismo sujeto califica de incorrecta. Así, en segmentos como el 3, la evasión no estaría relacionada con la falta de Moral tributaria, sino que el Moralista Evasor sería una especie de castigo al Estado ineficiente y corrupto que alienta a evadir sin culpa y a pesar de la presencia de una moral tributaria fuerte. Bajo ese contexto, la evasión no parece ser una acción condenada socialmente.

\section{III.3. La moral tributaria por segmento y las acciones para}

cada uno

¿De qué variables depende la moral de los individuos? La literatura económica suele establecer un conjunto de variables determinantes en la moral tributaria de las personas, como el sexo, los niveles de educación o su escala de ingreso, entre otras (una buena descripción de las variables determinantes de la moral se puede ver en Antequera y Florensa 2008). En este trabajo, donde se abordó la moral tributaria utilizando como aproximación de la misma la evaluación que realiza el individuo sobre la acción 'comprar en negro', encontramos algunos datos a considerar:

A) Se encontró una leve diferencia en la moral tributaria entre hombres y mujeres: mientras $72,4 \%$ de las mujeres consideraron como algo 'incorrecto' o 'más o menos incorrecto' comprar en negro, en el 
caso de los hombres ese porcentaje fue $67,3 \%$, es decir 5,1 puntos porcentuales menos.

B) La moral tributaria estaría más presente en grupos poblacionales con educación terciaria o universitaria (completa o incompleta) que en grupos poblacionales con educación primaria o secundaria. Mientras en el primer grupo el $66,2 \%$ de los encuestados mostró una Moral tributaria fuerte (MTF), en el segundo ese porcentaje se redujo a $54 \%, 12,2$ puntos porcentuales menos. A su vez, en la población que solo cuenta con instrucción primaria, el 36,4\% manifiesta ausencia de Moral tributaria (SMT) mientras que en los grupos con educación superior la proporción de personas SMT se reduce a solo $16,6 \%$ (promedio de universitarios y terciarios).

Moral tributaria según nivel de Instrucción

\begin{tabular}{lcrr}
\hline Estudios & MTF & MTD & \multicolumn{1}{c}{ SMT } \\
\hline Primarios & $54,5 \%$ & $9,1 \%$ & $36,4 \%$ \\
\hline Secundarios & $53,8 \%$ & $21,6 \%$ & $24,6 \%$ \\
\hline Terciarios & $66,2 \%$ & $18,2 \%$ & $15,6 \%$ \\
\hline Universitarios & $66,2 \%$ & $16,1 \%$ & $17,7 \%$ \\
\hline
\end{tabular}

C) Pero quizás la variable donde más marcadamente se presenta la diferencia de Moral tributaria, es la edad. Dividiendo la muestra por rangos de edades, se observa cómo a medida que aumenta la edad no solo se reduce la propensión a comprar en negro sino que se incrementa al mismo tiempo la moral tributaria. Por ejemplo, si entre los jóvenes de 18 a 25 años, el 67,3\% manifestó cierta moral tributaria (al considerar como incorrecto o más o menos incorrecto el pago de impuestos), entre la población mayor a 60 años el 95,1\% mostró cierta moral tributaria (MTF o MTD). De la misma manera, también la inconsistencia de la moral tributaria se reduce a mayor edad, aunque la diferencia entre estratos en este caso sea menos amplia que en el de la ausencia o presencia de moral tributaria.

La explicación de por qué a mayor edad mayor moral tributaria, podría ser que a medida que pasan los años la población se vuelve más consciente de lo que está bien y lo que está mal. Por su naturaleza, los jóvenes son más transgresores, y esa trasgresión abarca incluso acciones donde se presentan cuestiones éticas. A medida que pasan los años, el individuo va siendo más consciente de lo que implica 
ese acto. De la misma forma, la caída del porcentaje de moralistas corruptos en los segmentos poblacionales de mayor edad, también podría vincularse al hecho que la gente se vuelve más estricta con sus valores.

\begin{tabular}{lcccc}
\hline Edad & $\begin{array}{c}\text { Prefieren comprar en } \\
\text { negro para pagar } \\
\text { menos }(\$ 110)\end{array}$ & $\begin{array}{c}\text { Tienen cierta Moral } \\
\text { Tributaria (Fuerte o } \\
\text { Débil) }\end{array}$ & $\begin{array}{c}\text { Moralistas Evasores } \\
\text { (individuos con MTF que } \\
\text { elijen comprar en negro) }\end{array}$ & $\begin{array}{c}\text { Moralistas } \\
\text { Evasores } \\
\text { Conscientes }\end{array}$ \\
\hline $18-25$ & $62,0 \%$ & $67,3 \%$ & $51,9 \%$ & $53,8 \%$ \\
\hline $26-36$ & $60,4 \%$ & $78,5 \%$ & $49,0 \%$ & $73,9 \%$ \\
\hline $37-47$ & $57,5 \%$ & $83,1 \%$ & $47,8 \%$ & $75,0 \%$ \\
\hline $48-59$ & $55,2 \%$ & $86,3 \%$ & $45,7 \%$ & $75,0 \%$ \\
\hline mas 60 & $50,0 \%$ & $95,1 \%$ & $37,9 \%$ & $71,4 \%$ \\
\hline $\begin{array}{l}\text { Promedio de } \\
\text { la muestra }\end{array}$ & $\mathbf{5 8 , 6 \%}$ & $\mathbf{7 6 , 6 \%}$ & $\mathbf{4 3 , 5 \%}$ & $\mathbf{7 3 , 4 \%}$ \\
\hline
\end{tabular}

La diferenciación de segmentos poblacionales de acuerdo al grado de moral tributaria (segmentos con MTF, MTD y SMT) y la identificación de las características particulares de la población que integra cada uno de esos tres grupos, permite focalizar mejor las políticas tributarias dirigidas a cada uno de ellos para combatir la evasión. Si bien las políticas dependerán de qué tipo de tributo se busque controlar, en el caso puntual de los impuestos al consumo que es el que se analizó en este trabajo, donde el consumidor puede ser un controlador indirecto (si solicitara el ticket) y un evitador directo de la evasión (si no accediera a comprar en negro), las políticas deben orientarse a reforzar esos dos roles, esto es, el del individuo como controlador y como evitador al mismo tiempo de la venta informal. Por ejemplo, en el segmento sin moral tributaria integrado por un $23,4 \%$ de la población relevada, sería fundamental introducir valores que permitan informar a la población acerca del sistema tributario, qué es, por qué es importante pagar los impuestos, qué consecuencias tiene comprar en negro, cómo saber si se está comprando en condiciones de informalidad, etc. Aquí hay mucho que hacer desde la educación para generar una cultura tributaria y crear la moral tributaria, ausente en ese segmento. En muchos países, incluso en la Argentina, los organismos recaudadores han incorporado módulos de educación tributaria que buscan ese objetivo. Estos módulos a menudo se orientan a la población joven (alumnos primarios y secundarios), lo que es importante teniendo en cuenta que es dónde mayor predisposición a evadir se encuentra. Sí, como revelan las encuestas, la moral tributaria se incrementa casi naturalmente con el pasar de los años, las políticas educativas 
orientadas a la población joven, permitirían acelerar el proceso de afianzamiento de la moral tributaria.

En el caso del segmento con moral tributaria débil, donde el individuo califica el acto de comprar en negro como 'más o menos' incorrecto, se requieren una combinación de políticas. Por un lado, la cultura tributaria que afiance el concepto de evasión como algo perjudicial tanto para el individuo como para la sociedad. En segundo lugar, las políticas de estímulos (premios) que instalen el hábito de solicitar el ticket pueden ser un impulso indirecto. Por ejemplo, de la encuesta realizada surge que el $60 \%$ de los encuestados señalan que si el gobierno premiara por pedir el ticket lo solicitarían con mayor frecuencia. Sin embargo, no todos los premios parecen funcionar. En la Argentina, se implementó poco tiempo atrás el programa IVA y Vuelta donde el consumidor tiene la posibilidad de obtener un premio si solicita sus facturas. Como resultado, si bien $60 \%$ señala que pediría más el ticket si hay premio, solo $11 \%$ lo pide más que antes para participar de ese programa.

¿Qué pasa con el segmento 3, donde la moral tributaria es fuerte? Aquí la tarea es trabajar en el Moralista Evasor, quien evade a pesar de contar con una moral tributaria fuerte. Posiblemente aquí las políticas sean más dificultosas, ya que en el caso puntual de los impuestos al consumo, el 97,8\% de los Moralistas Evasores señalan que si el Estado fuera menos corrupto evitarían comprar en negro y estarían dispuestos a pagar todos sus tributos.

\section{DESENMASCARANDO LA MORAL}

Los estudios de moral tributaria constituyen un área donde queda un gran camino por desarrollar e investigar. Por ahora, son muchas las preguntas e incógnitas pendientes y relativamente escaso el conocimiento consolidado. Sabemos que la existencia de moral tributaria es una condición necesaria para reducir la evasión, e incrementar el cumplimiento fiscal. Pero al mismo tiempo, la evidencia para la Argentina muestra que en determinadas condiciones, la evasión puede coexistir con niveles elevados de moral tributaria.

Allí es donde a primera vista aparecen inconsistencias en la moral tributaria. La moral puede estar presente y no incidir en la decisión de pagar o no pagar del contribuyente, o en su decisión de comprar formal o informalmente, como se esperaría. La pregunta obvia 
es: ¿por qué ocurre eso? ¿Por qué una misma persona o grupo de personas pueden valorar como algo malo o incorrecto el no pago de impuestos, la evasión o el acto de comprar en negro, autodefinirse simultáneamente como un individuo que se comporta éticamente en la vida, pero al mismo tiempo evadir y no sentir culpa por hacerlo?

En mayor o menor medida la moral tributaria es un valor presente en todas las economías del mundo y eso explica por qué siempre existen individuos dispuestos a cumplir con las normas fiscales independientemente de todo tipo de variables que puedan alentar la evasión. Pero para buscar mecanismos eficientes para reducir la evasión, una de las primeras cuestiones que se deberían analizar, es 1) la intensidad con que se presenta esa moral en cada país; y 2) la consistencia de la misma. Si la moral solo abarca a un grupo minoritario de la población, y prevalece el segmento 1 (SMT), entonces hay que trabajar intensamente en la incorporación de la cultura y educación tributaria. Esa parecería ser una tarea de largo plazo, pero simple de llevar adelante. El problema mayor es cuando aparecen inconsistencias en la moral, esto es, una sociedad donde prevalece el segmento 3 (MTF) pero conformado por un grupo de moralistas dispuestos a evadir (Moralistas Evasores).

Aquí se presenta una limitación clara de la moral tributaria como guía del comportamiento fiscal. La pregunta a responder es: ¿por qué surgen los moralistas evasores? ¿Cómo contenerlos? Diversas causas explicarían la entrada en escena del Moralista Evasor. Algunas de ellas, son:

1. Problemas de Ingresos: frente a restricciones de ingresos, es común que muchos ciudadanos se vean obligados a violar sus propios valores morales. Aparecen así comportamientos irracionales o condenados por el propio individuo como dejar de para impuestos o comprar en el mercado informal. En este caso, el Moralista Evasor surge como consecuencia de problemas en la distribución de ingresos de la economía, pero es un rótulo que posiblemente le pese en su esquema de convicciones.

2. Percepciones de Ingresos: en sociedades con elevada percepción de pobreza y problemas de distribución de la riqueza como la Argentina, se puede esperar que el Moralista Evasor surja como en el caso anterior, solo que tratando de preservar cierto status de ingresos que resulta compatible con el deseado por el individuo. La mala distribución de la riqueza o la necesidad de acceder a un 
conjunto de bienes y servicios que el ciudadano considera indispensables para su vida, impulsan a comportarse en contra de sus valores, evadiendo.

3. Auto-ajuste de distorsiones del mercado: frente a distorsiones económicas o ciertas ineficiencias del mercado el individuo viola su moral tributaria auto justificándose su conducta en ellos. El ejemplo más común es la inflación, pero también hay moralistas evasores que fundamentan su conducta en un mercado que crea la necesidad de un determinado bien o servicio y luego lo hace privativo para el resto de la sociedad.

4. Percepción de corrupción o ineficiencia en la provisión de servicios del Estado: en la medida que el moralista percibe que el Estado es corrupto y la provisión de bienes y servicios para los que paga sus impuestos, es ineficiente, se convierte, sin culpa, en moralista evasor.

5. Ambición económica: es el caso típico de individuos con elevados niveles de riqueza que evaden para seguir incrementándola. Si bien se declaran con una moral tributaria fuerte, en su comportamiento cotidiano sus intereses económicos terminan pesando más que sus creencias o valores morales.

No sorprende que en sociedades con elevados niveles de evasión, generalmente esa informalidad responda en buena medida a problemas de ingresos, siendo este factor una de las causas más mencionadas por quienes evaden directa o indirectamente. El mayor problema sin embargo, se presenta cuando claramente las restricciones de ingresos ya distorsionaron la moral tributaria, de manera que la moral tributaria se adaptó a esa situación. En se esquema, acciones como 'comprar en negro' o directamente 'evadir' pueden seguir siendo consideradas como algo incorrecto por la sociedad, pero la sociedad no las condena, instalándose una especie de pacto implícito social donde una acción incorrecta es aceptada y en silencio, justificada.

Un comentario especial merece la relación entre los contribuyentes y el Estado. Los resultados de las encuestas indican que si el Estado fuera más transparente en el uso del gasto público, la sociedad tendría mayor predisposición al pago de impuestos. Al menos así lo declara el $60 \%$ de los entrevistados. Sin embargo, en la relación "Sociedad-Estado" es importante dejar planteados dos interrogantes: a) ila moral de la 
Sociedad (en sentido amplio) es muy diferente a la moral de los políticos que representan a los ciudadanos? De ser así, es decir, de existir dos tipos de moral diferentes, b) existe una relación de causalidad entre ambas: ¿la moral de los ciudadanos está influenciada por la moral de los gobernantes? o ila moral de la Sociedad se configura de manera independiente a la moral del Estado y, de hecho, la primera es la base esencial de la segunda? Independientemente de la respuesta a estos interrogantes, es crucial modificar los hábitos tributarios para las próximas generaciones y eso obliga a activar un conjunto de acciones combinadas que incrementen la moral tributaria, por un lado, pero generen condiciones adecuadas, por otro, para que el moralista no se convierta en evasor. Porque cuando eso ocurre, combatir la evasión se transforma en una tarea ardua y compleja, que requiere cambios estructurales que no siempre el Estado está en condiciones de implementar.

\section{BIBLIOGRAFÍA}

AitKen, S. and L. BonNeVILle (1980) A General Taxpayer Opinion Survey. Washington, DC: Internal Revenue Service.

AlLingham, M. and A. SANDmo (1972) "Income Tax Evasion: A Theoretical Analysis", Journal of Public Economics, 1(3/4), pp. 323-338.

ALM, J. (1999) "Tax Compliance and Administration". W.B. HILDRETH, J.A. RICHARDSON (eds.) Handbook on Taxation. New York: Marcel Dekker, pp. 741-768.

-, G. MCClelland and W. SChulze (1992) "Why Do People Pay Taxes?", Journal of Public Economics, 48(1), pp. 21-38.

-, G.H. MCCLELLAND and W.D. SCHULZE (1999) "Changing the Social Norm of Tax Compliance by Voting", KYKLOS, 48, pp. 141-171.

- and B. TORGLER (2006) "Culture Differences and Tax Morale in the United States and in Europe", Journal of Economic Psychology, 27, pp. 224-246.

ANDREONI, J.; B. ERARD and J. FEInSTEIn (1998) "Tax Compliance", Journal of Economic Literature, 36(June), pp. 818-860.

ANTEQUERA, Germán y Marcelo FLORENSA (2008) Determinantes de la Moral Tributaria en la Provincia de Buenos Aires. Serie Anales de la Asociación de Economía Política, Noviembre. 
Bosco, L. and L. MITTONE (1997) "Tax Evasion and Moral Constraints: Some Experimental Evidence", Kyklos, 50, 3, pp. 297-324.

CHUNG, P. (1976) "On Complaints About High Taxes, An Analytical Note", Public Finance, 31, pp. 36-47.

COWELL, F. and J. GORDON (1988) "Unwillingness to Pay", Journal of Public Economics, 36(3), pp. 305-321.

CULLIS, J. and A. LEWIS (1997) "Why People Pay Taxes: From a Conventional Economic Model to a Model of Social Convention", Journal of Economic Psychology, 18(2-3), pp. 305-321.

ERARD, B. and J. FEINSTEIN (1994) "The Role of Moral Sentiments and Audit Perceptions in Tax Compliance", Public Finance, 49 (Suppl.), pp. 70-89.

FREY, B.S. (2003) "Deterrence and Tax Morale in the European Union", European Revue, 11, 3, pp. 385-406.

FreY, R.L. and B. TORgLeR (2002) "Entwicklung und Stand der Steuermoralforschung", WiSt, 3, pp. 130-135.

GRAetZ, M.J. and L.L. WILDE (1985) "The Economics of Tax Compliance: Facts and Fantasy", National Tax Journal, 38, pp. 355-363.

Kantona, G. (1975) Psychological Economics. Amsterdam: Elsevier.

Lederman, L. (2003) "The Interplay Between Norms and Enforcement in Tax Compliance", Ohio State Law Journal, 64, 6, pp. 1453-1514.

LEWIS, A. (1971) Shame and Guilt in Neurosis. New York: International University Press.

- (1982) The Psychology of Taxation. Oxford: Martin Robertson.

Pommerehne, W.; A. HART and B. FreY (1994) "Tax Morale, Tax Evasion and The Choice of Policy Instrument in Different Political Systems", Public Finance, 49(Suppl.), pp. 52-69.

Roth, J.A.; J.T. SCHOLZ and A.D. WITTE (eds.) (1989) Taxpayer Compliance, 2 vols. Philadelphia: University of Pennsylvania Press.

SCHMÖLDERS, G. (1960) Das Irrationale in der öffentlichen Finanzwirtschaft. Probleme der Finanzpsychologie. Hamburg: Rowohlt.

- (1962) Teoría general del impuesto. Madrid: Editorial de Derecho Financiero.

- (1970) "Survey Research in Public Finance - A Behavioural Approach to Fiscal Theory", Public Finance, 25, pp. 300-306.

SonG, Y. \& T.E. Yarbrought (1978) "Tax Ethics and Taxpayer Attitudes: A Survey", Public Administration Review, 38, pp. 442-452. 
SPICER, M.W. and S.B. LUNDSTEDT (1976) "Understanding Tax Evasion", Public Finance, 31, pp. 295-305.

SUGDEN, R. (1984) "Reciprocity: the Supply of Public Goods through Voluntary Contributions", Economic Journal, 94, pp. 772-787.

TORGLER, B. (2003) "To Evade Taxes or not to Evade: That is The Question", Journal of Socio-Economics, 32(3), pp. 283-302.

- (2004) "Tax Morale in Asian Countries", Journal of Asian Economics, 15(2), pp. 237- 266.

- (2005) "Tax Morale in Latin America", Public Choice, 122(1-2), pp. 133157.

- and J. MARTINEZ (2005) The Evolution of Tax Morale in Modern Spain. Georgia State University: Andrew Young School of Policy Studies.

VIHANTO, M. (2003) "Tax Evasion and The Psychology of The Social Contract", Journal of Socio-Economics, 32(2), pp. 111-125.

WECK, H. (1983) Schattenwirtschaft: Eine Möglichkeit zur Einschränkung der öffentlichen Verwaltung?. Bern: Verlag Peter Lang Publishing Company. 